\title{
Documentos
}

\section{ACTUALIZACIÓN DE LA TERAPIA DEL HIRSUTISMO}

\author{
Enzo Devoto C. ${ }^{1}$, Lucía Aravena $C .^{1}$ \\ ${ }^{1}$ Facultad de Medicina, Universidad de Chile, Campus Central, Servicio de Medicina, Unidad de Endocrinología, Hospital \\ Clínico San Borja-Arriarán.
}

\section{RESUMEN}

El hirsutismo es un síntoma del hiperandrogenismo femenino a nivel dermatológico y constituye en sí mismo un problema estético y psicosocial para la mujer. Se analizan las drogas involucradas en el tratamiento, el mecanismo de acción y efectos colaterales. La eficiencia y seguridad de ciproterona, espironolactona y flutamida en 3 grupos de mujeres hirsutas se comparan con los datos de la literatura.

\section{PALABRAS CLAVES: Hirsutismo, antiandrógenos, tratamiento}

\section{SUMMARY}

The hirsutism is a symptom of the feminine hiperandrogenism at dermatological level and constitutes in itself an aesthetic and psycho-social problem for the woman. The drugs involved in the treatment, the mechanism of action and the collateral effects are analyzed. The efficiency and security of cyproterone, spironolactone and flutamide in 3 groups of hirsute women are comparing with the literature data.

\section{KEY WORDS: Hirsutism, antiandrogens, treatment}

\section{INTRODUCCIÓN}

El hiperandrogenísmo femenino se manifiesta a nivel dermatológico como hirsutismo, acné, seborrea y alopecia androgénica. Se debe al aumento de la producción de andrógenos ováricos y/ o suprarrenales, o a un fenómeno periférico en que andrógenos plasmáticos normales en una mujer con función ovárica normal (hirsutismo idiopático o periférico) pueden producir un similar grado de hirsutismo.

El hirsutismo periférico se debería al aumento de la actividad de la 5 alfa reductasa, aumento de la IGF1, disminución de la IGF BP1 o cambios en el receptor androgénico (RA) $(1,2)$. Diagnosticada la causa del hiperandrogenismo, se realiza un tratamiento integral, metabólico y nutricional, siendo el enfrentamiento del hirsutismo un elemento más del enfoque terapéutico.

\section{TERAPIA MÉDICA DEL HIRSUTISMO}

El tratamiento del hirsutismo consiste en: 1) frenación de la secreción de andrógenos, 2) antiandrógenos (AA), 3) inhibidores de la 5 alfa reductasa, 4) insulinosensibilizadores.

\section{Frenación de la secreción de andrógenos}

1a) Anticonceptivos (ACO). La combinación de estrógeno y progestágeno antiandrogénico (clormadinona y drosperidona) o androgénicamente neutro (gestodeno, desogestrel, norgestimato) tiene por objeto: a) Frenar LH, disminuyendo el estímulo sobre el componente tecal del ovario y bajando los niveles de andrógenos; b) Incrementar la síntesis de SHBG y disminuir la testosterona libre; c) Frenar el componente androgénico suprarrenal; d) Producir la maduración endometrial, en los 
hiperandrogenísmos con anovulación crónica, disminuyendo el riesgo de metrorragia, anemia y cáncer de endometrio; e) Algunos progestágenos inhibirían la 5 alfa reductasa de la piel y otros como la clormadinona y drosperidona competirían con el andrógeno en su receptor (3).

El uso de ACO no constituye una terapia curativa. Al suspenderlos generalmente recidivará el hiperandrogenismo y la anovulación. Son útiles para ser asociados con algún AA, evitando embarazo y el déficit de virilización de un feto masculino expuesto a dichos fármacos, o a los inhibidores de la 5 alfa reductasa. Impiden los trastornos menstruales que puede producir la espironolactona. Debe vigilarse la aparición de disminución de la sensibilidad insulínica y alteración del perfil lípidico.

Existen dos posiciones con respecto al uso de ACO en la terapia del síndrome de ovario poliquístico (SOP). Una la considera terapia de primera línea que debe ser mantenida crónicamente mientras la paciente no desee fertilidad, minimizando a nuestro entender las complicaciones metabólicas (4) y otra, que sin negar los efectos beneficiosos de los ACO en la terapia, alerta respecto al efecto deletéreo del incremento de la resistencia insulínica sobre las complicaciones a largo plazo, como diabetes tipo 2 y enfermedad cardiovascular (5). El efecto del ACO puro en el control del hirsutismo es variable; algunas experiencias informan la disminución de este $(6,7,8)$ y otras en que el efecto es muy leve (menor del 10\%) o inexistente (1).

Se ha planteado un efecto aditivo al combinar AA más ACO como demostraron Gregorieu y cols (7) al indicar espironolactona con etinilestradioldesogestrel; nosotros utilizamos este mismo ACO más flutamida sin poder demostrar ese efecto aditivo (9). La falta del efecto aditivo que generaría el ACO, cuestiona la importancia de la frenación de andrógenos, del aumento de la SHBG y disminución de la testosterona libre, en su significación clínica a nivel periférico para reducir el hirsutismo, hecho que ha sido compartido por otros autores $(10,11)$.

1b) Frenación de la secreción de andrógenos por glucocorticoides. Existe un hiperandrogenísmo glucocorticoide supresible que comprende: a) hiperandrogenismo suprarrenal de base genética por déficit enzimáticos de la 21 hidroxilasa, 11 hidroxilasa y 3 beta ol dehidrogenasa, en su forma congénita y la de inicio tardío post puberal, y b) hiperandrogenismo funcional suprarrenal (HFS) no debido a defectos enzimáticos de base genética, donde el glucocorticoide actuaría igual que en el caso anterior, inhibiendo centralmente $\mathrm{CRH}$ y
ACTH, y además en la reticular modificando interleukinas y $\mathrm{CRH}$ producidos localmente. Se ha señalado que los glucocorticoides también pueden frenar andrógenos ováricos (12-18).

Al suspender los glucocorticoides en el HFS existe un período prolongado de remisión con escasas recaídas, lo que no ocurre en los de base genética (19). El glucocorticoide disminuye modestamente el hirsutismo (20\%), pese al descenso de los niveles de andrógenos (20).

Se ha planteado la conveniencia de la frenación de la fuente androgénica con glucocorticoides, más la administración de AA en terapia de hirsutismo, lo que en nuestra experiencia (21) y la de otros autores (20) no se demostró. Aunque se frene con glucocorticoides los andrógenos ováricos en un subgrupo de SOP, actualmente su uso crónico está contraindicado ya que el alza de peso y el incremento de la resistencia insulínica por ellos generados, podría favorecer las complicaciones crónicas de esta enfermedad (22). Su uso se reserva para los déficit enzimáticos suprarrenales de base genética y posiblemente en el HFS por un período de 1 o 2 años, esperando obtener remisión post suspensión. Se ha señalado que un AA periférico puede ser más eficaz que el glucocorticoide, para tratar el hirsutismo en el síndrome adrenogenital genético de inicio tardío (23).

1c) Inhibición de la síntesis de andrógenos por ketoconazol. Este disminuye la síntesis de testosterona y cortisol mediante inhibición de la 17 hidroxilasa y 17-20 lyasa, con un efecto dosis dependiente (24). Con dosis de $200 \mathrm{mg} 2$ veces al día se ha logrado disminución de los andrógenos $y$ un descenso del hirsutismo menor que el obtenido con otros AA. Debe considerarse la posibilidad de hepatotoxicidad severa; a mayor dosis generan insuficiencia suprarrenal e hipocalcemia por inhibición de enzimas renales involucradas en el metabolísmo de la vitamina $\mathrm{D}$.

1d) Análogos del GNRH. La frenación de la secreción de andrógenos ováricos se obtiene por la producción de un hipogonadismo hipogonadotropo debido a la acción hipofisiaria de los análogos del GNRH. Concomitantemente con la frenación de andrógenos se produce un hipoestrogenismo y disminución de masa ósea; estos efectos y su alto costo limitan su utilización en la terapia del hiperandrogenismo.

\section{Antiandrógenos}

Dorfman en 1970 definió a los AA como sustancias que impiden la acción del andrógeno en 
su efector. Este efecto es diferente al que logran las sustancias que frenan el eje hipotálamo-hipófisisovario (análogos de GNRH, ACO, medroxiprogesterona) y de las que inhiben enzimas que intervienen en la síntesis de andrógenos (ketoconazol). Según la estructura química se diferencian en: esteroidales (ciproterona, espironolactona) y no esteroidales (flutamida). Según su mecanismo de acción es el bloqueo del receptor de andrógenos (RA) más otros efectos endocrinos (ciproterona, espironolactona) y antiandrógeno puro (flutamida) (25).

2a) Acetato de ciproterona. Mecanismos de acción: a) competición con el andrógeno a nivel del RA; b) antigonadotropo; c) acción progestativa potente; d) inhibición de la 5 alfa reductasa; e) frenación de ACTH por efecto glucocorticoideo al ser administrada en altas dosis.

Esquemas terapéuticos: a) terapia combinada: ciproterona $2 \mathrm{mg}$ más etinilestradiol 0,035 mg por 21 días; b) esquema secuencial reverso: etinilestradiol $0,035 \mathrm{mg}$ por 21 días más ciproterona 50 a $100 \mathrm{mg}$ del día 1 al 10 del ciclo; c) ciproterona 2 mg más etinilestradiol 0,035 por 21 días más 10 mg de ciproterona del día 1 al 10 del ciclo (26). Se indica preferentemente el esquema combinado en la terapia de hirsutismo, acné y seborrea; el esquema secuencial reverso y la suplementación de la terapia combinada con dosis variable de ciproterona se utilizan en hiperandrogenismos severos, aunque aumenta el costo.

Efectos colaterales: mamarios (mastalgia y aumento de volumen), retención hídrica y aumento de peso, síntomas depresivos $(27,28)$. Como todo AA puede disminuir la líbido, efecto que es reversible. Existe la posibilidad que genere o incremente la insulinorresistencia por lo que en SOP se recomienda utilizarla con insulinosensibilizadores.

2b) Espironolactona. Mecanismos de acción: a) disminuye la síntesis de andrógenos por inhibición de la 17 hidroxilasa; b) efecto progestativo que explicaría las alteraciones menstruales; c) efecto antiandrogénico, se liga competitivamente al RA. Estudios in vitro demostraron que esta acción es más potente que la de otros $A A$, pero in vivo su metabolito la canrenona, tiene vida media corta lo que disminuye su acción clínica. A nivel del RA tiene efecto agonísta - antagonísta lo que explicaría la agravación de acné y seborrea que puede ocurrir al inicio de la terapia (29-31). La mayoría de los trabajos no demuestran descensos de los niveles de andrógenos al dar espironolactona, lo que indicaría que los resultados experi- mentales de disminuir la síntesis de andrógenos no tienen gran importancia clínica.

Efectos colaterales: aumento de diuresis y fatigabilidad en los primeros días de terapia y alteraciones del ciclo menstrual, según algunos muy frecuentes (32) y según otros, poco frecuentes y con excelente tolerancia (33). Debe vigilarse riesgo de hiperkalemia.

2c) Flutamida. Mecanismos de acción: Es un AA no esteroidal desprovisto de acción endocrina. Este último punto no es absoluto ya que disminuye la DHEA-S por inhibición de la 17 hidroxilasa, 17-20 lyasa o por disminución del clearance de cortisol, produciéndose un aumento del cortisol que frena ACTH, lo que inhibe la síntesis androgénica de la reticular suprarrenal (34-36). In vitro es superada por la espironolactona en la competición por el RA; in vivo su metabolito, la 2 hidroxiflutamida es más potente por poseer una vida media más larga, la que aumenta al incrementar la dosis. Carece de efecto agonísta en el RA, no tiene actividad estrogénica, progestativa ni glucocorticoidea.

Efectos colaterales: hepatotoxicidad, lo que implica estudio de función hepática pre terapia y durante su administración (37).

\section{Inhibidores de la 5 alfa reductasa}

Finasteride. Es un inhibidor de la 5 alfa reductasa tipo 2, enzima mayoritariamente presente en próstata y tejidos genitales que transforma la testosterona en dihidrotestosterona, compuesto de mayor potencia que el anterior. Si bien la enzima más importante a nivel cutáneo parece ser la 5 alfa reductasa tipo 1 , el finasteride ha logrado disminución de hirsutismo y discutible acción en acné (38).

\section{Insulinosensibilizadores}

Son agentes que aumentando la sensibilidad del tejido efector a la insulina disminuyen la resistencia a esta hormona, por lo cual baja la hiperinsulinemia compensadora.

4a) Metformina. El mecanismo de acción aún es discutido, parece ser importante la disminución de la producción hepática de glucosa por inhibición de la gluconeogénesis y glucogenolisis. También podría inhibir la absorción intestinal de glucosa y aumentar su captación y utilización en músculo y otros tejidos (39). La metformina influiría en la producción de hormonas generadas en el tejido adiposo (adipoquinas), diminuyendo las pro infla- 
matorias, aterogénicas y generadoras de resistencia insulínica, y favoreciendo el alza de las antiinflamatorias e insulinosensibilizadoras como la adiponectina (40). En dosis de $850 \mathrm{mg} 2$ veces al día administrada en SOP con resistencia insulínica e hiperinsulinemia, mejora la sensibilidad a la insulina, logra eumenorrea y ovulación. En algunas pacientes disminuye los andrógenos plasmáticos y en otras no hay cambios. Se ha explicado el descenso de los andrógenos por disminución del hiperinsulinismo en que cesaría la acción conjunta de LH e insulina sobre la célula tecal (41). Otra vía de acción es la posible inhibición de las enzimas que favorecen la esteroidogénesis ovárica y adrenal de andrógenos (42). Un autor reporta importante disminución del hirsutismo al ser administrada sin AA (41); la mayoría concuerda en que su efecto sobre hirsutismo es clínicamente poco relevante $(43,44)$. En adolescentes sería recomendable asociar metformina con un AA, obteniéndose mejoría del hirsutismo con protección metabólica (45). De ser necesario agregar un ACO se recomienda que contenga como progestágeno drosperidona, cuya acción es más parecida a la progesterona natural y vinculada químicamente y funcionalmente con la espironolactona (46).

4b) Tiazolidinedionas. Son ligandos del PPAR gama (receptor activado de proliferación de peroxisomas); éstos receptores pertenecen a la familia de los receptores nucleares y actúan en la transcripción genética. Las tiazolidinedionas se ligan al PPAR gama activándolo y éste actúa sobre elementos de respuesta en ciertos genes que codifican proteínas que regulan el metabolismo lipídico y la acción insulínica. Aumentan la sensibilidad a la insulina en músculo y tejido adiposo, disminuyendo la producción de ácidos grasos libres y adipoquinas proinflamatorias provenientes de la grasa abdominal (47). La primera representante de esta familia, la troglitazona, retirada del merca- do por su hepatotoxicidad demostró mejorías metabólicas y reproductivas en SOP. Azziz y cols demostraron un $15 \%$ de disminución del score de hirsutismo a las 44 semanas de terapia (48).

Las nuevas tiazolidinedionas, rosiglitazona y pioglitazona utilizadas en SOP, disminuirían la producción de andrógenos, regularían el ciclo menstrual y la frecuencia ovulatoria (49). Superarían a metformina en inducir respuesta ovulatoria al ser administradas solas (50).

En pacientes con SOP que desean fertilidad y no se produce ovulación bajo metformina y tiazolidinedionas, se pueden combinar con citrato de clomifeno; esta combinación se ha demostrado útil incluso en pacientes que no responden al clomifeno solo (clomifeno resistentes), siendo la mezcla clomifeno con tiazolidinedionas más efectiva que clomifeno con metformina (50).

Su mecanismo de acción se ejerce a través del descenso de la insulinemia y un efecto inhibidor sobre las enzimas de la biosíntesis androgénica ovárica y suprarrenal (51). En terapia del hirsutismo producido por SOP se han asociados las tiazolidinedionas con etinilestradiol-ciproterona, disminuyendo la insulinoresistencia, los andrógenos y mejorando el hirsutismo, similar a lo que otro autor ha demostrado con la suplementación de metformina a etinilestradiol-ciproterona $(52,53)$. Hay datos aún controvertidos respecto al descenso de los andrógenos y porcentaje de mejoría del hirsutismo con pioglitazona y rosiglitazona, ya que los grupos estudiados son pequeños y la duración del tratamiento es breve.

\section{EFICIENCIA Y SEGURIDAD DE LOS ANTIANDRÓGENOS EN TERAPIA DE HIRSUTISMO}

La Tabla I señala nuestra experiencia en eficacia terapéutica con 3 grupos de AA: esquema

Tabla I

DISMINUCIÓN DEL PROMEDIO DEL SCORE DE MONCADA - LORENZO* EN HIRSUTAS TRATADAS CON 3 TIPOS DE ANTIANDRÓGENOS (CIPROTERONA/ETINILESTRADIOL - ESPIRONOLACTONA - FLUTAMIDA)

\begin{tabular}{lccc}
\hline Tratamiento & EE $0,035 \mathrm{mg}+$ ciproterona $2 \mathrm{mg} / \mathrm{dí} a$ & Espironolactona $100 \mathrm{mg} /$ día & Flutamida $500 \mathrm{mg} /$ día \\
$n$ & 74 & 54 & 43 \\
\hline Puntaje basal & $11,2 \pm 3,5$ & $11,5 \pm 2,1$ & $11,5 \pm 2,7$ \\
Puntaje 6 meses & $7,1 \pm 2,1$ & $6,5 \pm 2,5$ & $7,0 \pm 2,4$ \\
Puntaje 12 meses & $4,3 \pm 2,7$ & $6,0 \pm 2,1$ & $5,0 \pm 2,5$ \\
\hline
\end{tabular}

EE: etinilestradiol.

p basal (etnilestradiol + ciproterona vs espironolactona vs flutamida) $=0,81$ (NS).

p 12 meses (etinilestradiol + ciproterona vs espironolactona vs flutamida) $=0,25$ (NS).

*Moncada E. Familial study of hirsutism. J Clin Endocrinol Metab 1970;31:691-5. 
combinado continuo de ciproterona (etinilestradiol $0,035 \mathrm{mg}$ + ciproterona $2 \mathrm{mg}$ ), espironolactona $100 \mathrm{mg} /$ día y flutamida $500 \mathrm{mg} / \mathrm{día}$. La disminución del score basal vs el score al año de terapia, revelan una respuesta similar, lo que concuerda con varios trabajos que no demuestran diferencia importante de la respuesta clínica entre los distintos AA $(54,55,56)$, a diferencia de otros que obtienen una mayor potencia terapéutica con la utilización de flutamida $(57,58)$.

Los mecanismos de acción de los AA parecen no ser importantes para el efecto sobre el hirsutismo, ya que Fruzzetti y cols encuentran que finasteride, ciproterona y flutamida son igualmente efectivos en disminuirlo, pese a tener diferentes mecanismos de acción (57).

Referente a la seguridad de estos fármacos, los efectos colaterales en las pacientes sometidas a la combinación $2 \mathrm{mg}$ de ciproterona $+35 \mu \mathrm{g}$ de etinilestradiol fueron molestias a nivel mamario, aumento de peso y trastornos del ánimo. Esto concuerda con lo publicado en un estudio cooperativo alemán (27) y con la experiencia previa de los autores (59).

La literatura señala un alto porcentaje de trastornos menstruales al administrar espironolactona sola, esto fue poco frecuente en nuestra casuística (13,3\% de goteo) (21) que no motivó interrupción de tratamiento; las pacientes solamente refirieron poliuria, fatigabilidad y agravación del acné en los inicios de la terapia. No hubo casos de hiperkalemia ya que fueron criterio de exclusión los siguientes antecedentes: nefropatías, recibir suplementos de potasio y medicamentos que alteren su metabolísmo (AINES, diuréticos ahorradores de potasio, antihipertensivos del tipo bloqueadores de la enzima convertidora y del receptor de angiotensina). Las pacientes tratadas con flutamida no presentaron síntomas colaterales importantes ni cambios en la función hepática, lo que en ningún caso justifica que se ignore la posibilidad de hepatotoxicidad grave.

\section{BIBLIOGRAFÍA}

1. Rittmaster RS. Treatment of hirsutism. Endocrinologist 1993;3:211-8.

2. Azziz R, Carmina E. Idiopathic hirsutism: definition, prevalence and inheritance. Androgen excess disorders in women. Nestler GR, Dewailly D, Azziz R (Ed); Lippincott- Raven publisher Philadelphia 1997;529-34.

3. Azziz R, Gay F. The treatment of hyperandrogenic with oral contraceptives. Sem Reprod Endocrinol 1989;7:246-54.
4. Adams Hillard PJ. Oral contraceptives and the management of hyperandrogenism - polycystic ovary syndrome in adolescents. Endocrinol Metab Clin N Am 2005;34:677-705.

5. Diamanti-Kandarakis E, Mitrakou A, Hennes MM I, Platanissiotis D, Karlas N, Spina J, et al. Insulin sensitivity and antiandrogenic therapy in women with polycystic ovary syndrome. Metabolism 1995;44: 525-31.

6. Culberg G, Hamberger L, Mattson L, Mobacken H, Samsioe G. Effects of a low dose desogestrel ethinylestradiol combination on hirsutism, androgen and sex hormone binding globulin in women with a polycystic ovary syndrome. Acta Obstet Gynecol Scand 1985;64:195-202.

7. Gregoriou O, Bakas P, Konidaris S, Papadias K, Mathiopoulos D, Creatsas $G$. The effect of combined oral contraception with or without spironolactone on bone mineral density of hyperandrogenaemic women. Human Reproduction 1993;8:1807-12.

8. Porcile A, Gallardo E. Long-term treatment of hirsutism: desogestrel compared with cyproterone acetate in oral contraceptives. Fertil Steril 1991; 55:877-81.

9. Devoto E, Aravena L. Eficacia de la flutamida en el tratamiento del hirsutismo. Contribución del uso combinado con anticonceptivos orales en mujeres normoandrogénicas. Rev Méd Chile 2004;132:84552.

10. Elkind-Hirsh K E, Anania C, Mack M, Malinak R. GNRH agonist plus oral contraceptive - ad back therapy stimulate sex hormone binding globulin, and lowers free testosterone in hirsute women with ovarian hiperandrogenism. Fertil Steril 1995;63:9708.

11. Azziz R, Ochoa TM, Bradley EL, Downing Potter H, Boots LR. Leuprolide and estrogen versus oral contraceptive randomized study. J Clin Endocrinol Metab 1995;80: 3406-8.

12. Devoto E, Aravena L. Hiperandrogenismo suprarrenal funcional: perspectivas cambiantes en su evolución histórica. Rev Méd Chile 1995;123:101624.

13. Abraham GE, Maroulis GB, Boyers SP. Dexamethasone suppression test in the management of hyperandrogenized patients. Obstet Gynecol 1981;57:15865.

14. Steinberger E, Rodríguez - Rigau LJ, Petak SM. Glucocorticoid therapy in hyperandrogenism. Bailliére`s Clin Obstet Gynecol 1990;4:457-71.

15. Kirschner MA, Jacob JB. Combined ovarian and adrenal vein catheterization to determine the site(s) of androgen overproduction in hirsute women. J Clin Endocrinol Metab 1971;33:199-206.

16. Rosenfield RL, Ehrmann DA, Barnes RB. Gonadotropin- releasing hormone agonist as a probe for the pathogenesis and diagnosis of ovarian hyperandrogenism. Ann N Y Acad Sci 1993;687:162-77.

17. Buggs $C$, Rosenfield RL. Polycystic ovary syndrome 
in adolescence. Endocrinol Metab Clin North Am 2005;34:67-705.

18. Ehrhart-Bornstein M, Hinson JP, Bornstein SR, Scherbaum WA, Vinson GP. Intraadrenal interactions in the regulation of adrenocortical steroidogenesis Endocrin Rev 1998;19:101-43.

19. Devoto E, Aravena L, Gaete X. Remisión prolongada del hiperandrogenismo femenino después de suspender la terapia con glucocorticoides. Rev Méd Chile 1995;123:207-14.

20. Carmina E, Lobo R. Peripheral androgen blockade versus glandular androgen suppression in the treatment of hirsutism. Obstet Gynecol 1991;78:8459.

21. Devoto E, Aravena L, Ríos S. Tratamiento del hirsutismo con espironolactona y con espironolactona más dexametasona. Rev Méd Chile 2000;128:86875.

22. Azziz R, Black VY, Knochenhauer AS, Hines GA, Boots LR. Ovulation after glucocorticoid suppression of adrenal androgens in the basal dehydroepiandrosterone sulfate level. J Clin Endocrinol Metab 1999;84:946-50.

23. Spritzer P, Billaud L, Thalabard JC. Cyproterone acetate versus hydrocortisone treatment in late onset adrenal hyperplasia. J Clin Endocrinol Metab 1990;70:642-6.

24. Pepper G, Brenner SH, Gabrielove JL. Ketoconazole use in the treatment of ovarian hyperandrogenism. Fertil Steril 1990;54:438-44.

25. Devoto E, Aravena L. Utilidad terapéutica de los antiandrógenos. Rev Méd Chile 1993;121:1183-90.

26. Hammerstein J. Use of cyproterone acetate in the treatment of acne, hirsutism and virilism. J Steroid Biochem 1975;6:827-31.

27. Hammerstein J. Possibilities and limits of endocrine therapy. Androgenization in women, in Hammerstein J, Lachnit - Fixson U, Plewig G (Eds). Excerpta Médica 1980;222-38.

28. Breckwoldt M. Side-effects and contraindications of treatment with antiandrogens. Androgenization in women, in Hammerstein J, Lachnit - Fixson U, Plewig G (Eds). Excerpta Médica 1980:266-75.

29. Young RL, Goldzieher JW, Elkind - Hirsch K. The endocrine effects of spironolactone used as an antiandrogen. Fertil Steril 1987;48:223-8.

30. Eil Ch, Edelson SK. The use of human skin fibroblasts to obtain potency estimates of drug binding to androgen receptors. J Clin Endocrinol Metab 1984; 59:51-5.

31. Luthy IA, Begin DJ, Labrie F. Androgenic activity of synthetic progestins and spironolactone in androgensensitive mouse mammary carcinoma (shionogi) cells in culture. J Steroid Biochem 1988;31:845-52.

32. Helfer E, Miller JL, Rose LI. Side - effects of spironolactone therapy in the hirsute woman. J Clin Endocrinol Metab 1988;208-11.

33. Barth JH, Cherry CA, Wojnarowska F, Dawber PR. Spironolactone is an effective and well tolerated systemic antiandrogen therapy for hirsute women. J Clin Endocrinol Metab 1989;68:966-70.

34. Labrie F. Mechanism of action and pure antiandrogen properties of flutamide. Cancer 1993;72:381627.

35. Ayub M, Levell M J. Inhibition of rat testicular 17 hydroxylase and 17, 20 lyase activities by antiandrogens (flutamide, hydroxiflutamide, RU23908, cyproterone acetate) in vitro. J Steroid Biochem 1987;28:43-7.

36. Fukushima DK, Levin J, Krean J, Freed SZ, Whitmore WF, Hellman L, et al. Effect of flutamide on cortisol metabolism. J Clin Endocrinol Metab 1978; 47:788-91.

37. Wysowski DK, Fourcroy JL. Flutamide hepatotoxicity. J Urol 1996;155:209-12.

38. Fruzzetti F, De Lorenzo D, Parrini D. Effect of finasteride, a 5 alfa reductasa inhibitor, on circulating androgen and gonadotropin secretion in hirsute woman. J Clin Endocrinol Metab 1994;70:831-5.

39. Zimmet P, Collier G. Clinical efficacy of metformin against insulin resistance parameters. Drugs 1999; 58:21-8

40. Ibañez L, De Zegher F. Ethinylestradiol - drosperidona, flutamide - metformina or both for adolescents and women with hyperinsulinemic hyperandrogenism: opposite effects on adipocytokines and body adiposity. J Clin Endocrinol Metab 2004;89:1592-7.

41. Pasquali R, Gambineri A, Biscotti D, Vicennati V, Gagliardi L, Colitta D, et al. Effect of long - term treatment with metformin added to hypocaloric diet on body composition, fat distribution, and androgen and insulin levels in abdominal obese women with and without the polycystic ovary syndrome. J Clin Endocrinol Metab 2000;85:2767-74.

42. Ibañez L, Valls C, Potau N, Marcos M V, De Zegher F. Sensitization to insulin in adolescente girls to normalize hirsutism, hyperandrogenism, oligomenorrhea, dyslipidemia, and hyperinsulinism after precocious pubarche. J Clin Endocrinol Metab 2000;85: 3526-30.

43. Moghetti P, Castello R, Negri C, Tosi F, Perrone F, Caputto $M$, et al. Metformin effects on clinical features, endocrine and metabolic profiles, and insulin sensitivity in polycystic ovary syndrome: a randomized, double-blind, placebo-controlled 6 month trial, followed by open, long - term clinical evaluation. J Clin Endocrinol Metab 2000;85:139-46.

44. Morin - Papunen LC, Koivunen RM, Ruokonen A, Martikainen HK. Metformin therapy improves the menstrual pattern with minimal endocrine and metabolic effects in women with polycystic ovary syndrome. Fertil Steril 1998;69:691-6.

45. Ibañez L, Ong K, Ferrer A, Amin R, Dunger D, De Zegher F. J Clin Endocrinol Metab 2003;88:2600-6.

46. Batukan C, Muderris II. Efficacy of a new contraceptive containing drosperidone and ethinyl estradiol in the long - term treatment of hirsutism. Fertil Steril 2006;85:436-40. 
47. Uauy R, Martinez JI, Rojas CV. Nutrición molecular, papel del sistema PPAR en el metabolismo lipídico y su importancia en obesidad y diabetes mellitus. Rev Méd Chile 2000;128:437-46.

48. Azziz R, Ehrmann D, Legro RS, Whitcomb RW, Hanley R, Gmerek Fereshtian A, et al. Troglitazone improves ovulation and hirsutism in the polycystic ovary syndrome: a multicenter, double blind, placebo - controlled trial. J Clin Endocrinol Metab 2001;86: 1626-32.

49. Ortega - Gonzalez C, Luna S, Hernandez L, Crespo $\mathrm{G}$, Aguayo, et al. Responses of serum androgen and insulin resistance to metformin and pioglitazone in obese, insulin - resistant women with polycystic ovary syndrome. J Clin Endocrinol Metab 2005;90: 1360-5.

50. Rouzi AA, Ardawi SM. A randomized controlled trial of the efficacy of rosiglitazone and clomiphene citrate - resistant polycystic ovary syndrome. Fertil Steril 2006;85:428-35.

51. Yilmaz M, Karakoc A, Toruner FB, Cakir N, Tiras B, Ayvaz G, et al. The effects of rosiglitazone and metformin on menstrual cyclicity and hirsutism in polycystic ovary syndrome. Gynecol Endocrinol 2005;21:154-60.

52. Lemay A, Dodin S, Turcot L, Dechene F, Forest JC. Rosiglitazone and ethinyl estradiol/cyproterone acetate as single and combined treatment of overweight women with polycystic ovary syndrome and insulin resistance. Hum Reprod 2006;21(1):121-8.
53. Elter K, Imir G, Durmusoglu F. Clinical, endocrine and metabolic effects of metformin added to ethiny lestradiol - cyproterone acetate in non obese women with polycystic ovarian syndrome: a randomized controlled study. Hum Reprod 2002;17:1729-37.

54. Moghetti P, Tosi F, Tosti A, Negri C, Misciali C, Perrone $\mathrm{F}$, et al. Comparison of spironolactone, flutamide, and finasteride efficacy in the tratment of hirsutism:a randomized, double blind, placebo - controlled trial. J Clin Endocrinol Metab 2000;85:89-94.

55. Venturoli S, Marescalchi O, Colombo F M, Macrelli S, Ravaioli M B, Bagnoli A, et al. A prospective randomized trial comparing low dose flutamide, finasteride, ketoconazole, and cyproterone acetate estrogen regimens in the treatment of hirsutism. J Clin Endocrinol Metab 1999;84:1304-10.

56. Fruzzetti F, Bersi Ch, Parrini D, Cabiria R, Genazzani AR. Treatment of hirsutism: comparisons between different antiandrogens with central and peripheral effects. Fertil Steril 1999;71:445-51.

57. Cusan L, Tremblay R R, Dupont A, Labrie F, Gomez $\mathrm{JL}$. Comparison of flutamide and spironolactone in the treatment of hirsutism: a randomized controlled trial. Fertil Steril 1994;61:281-7.

58. Muderris I I, Bayram F, Guven M. A prospective, randomized trial comparing flutamide $(250 \mathrm{mg} / \mathrm{d})$ and finasteride $(5 \mathrm{mg} / \mathrm{d})$ in the treatment of hirsutism. Fertil Steril 2000;73:984-7.

59. Devoto E, Aravena L, Madariaga M. Utilidad de la terapia antirreceptor de hormonas sexuales en la mujer y el varón. Rev SOGIA 1995;2:16-22. 